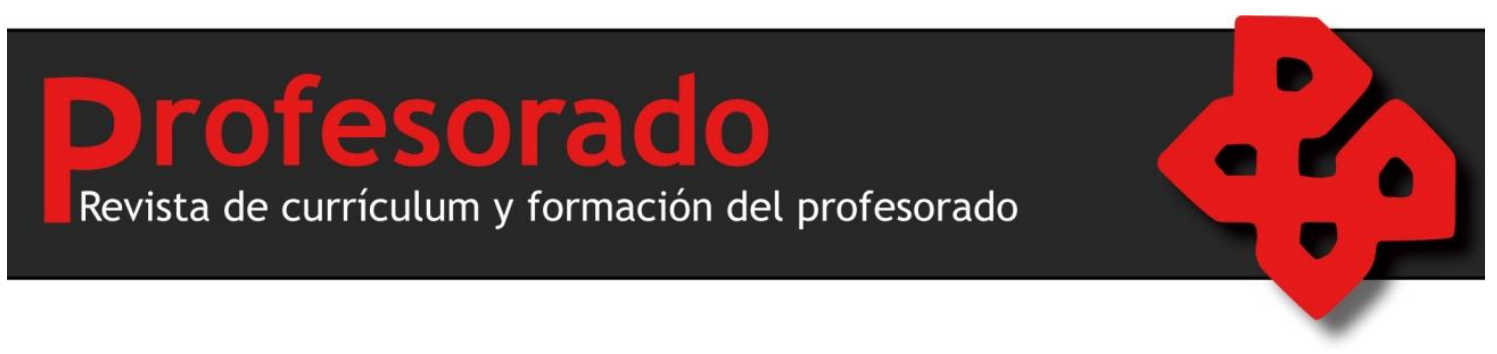

Vol. 23, № 3 (Julio-septiembre, 2019)

ISSN 1138-414X, ISSNe 1989-639X

DOI: $10.30827 /$ profesorado.v23i3.11239

Fecha de recepción: 10/04/2017

Fecha de aceptación: 09/11/2017

\title{
EL LUGAR DEL CURRÍCULUM EN LA PRIMERA INFANCIA: APORTES DE UNA MIRADA COMPARADA
}

The place of curriculum in early childhood education: insights from a comparative approach

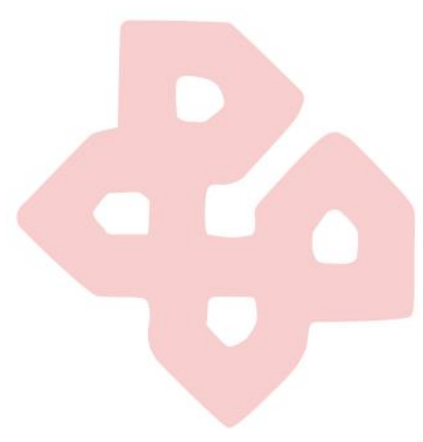

Jennifer Guevara

Alejandra Cardini

Centro de Implementación de Políticas Públicas

para la Equidad y el Crecimiento (Argentina)

E-mail: jguevara@cippec.net ; acardini@cippec.org

ORCID ID : https:// orcid.org/0000-0003-2581-5102

Resumen

La atención y educación de la primera infancia (AEPI) se ha convertido en una prioridad para las políticas educativas en distintos países de la región y del mundo. Su importancia estuvo acompañada por una progresiva institucionalización de la provisión y un mayor desarrollo curricular. Sin embargo, en muchos países persiste un panorama fragmentado, en los que la AEPI es provista bajo diversos formatos por instituciones con distintas rectorías. El trabajo examina la interacción de los principales documentos curriculares de seis países y una jurisdicción con las características del mapa de provisión educativa en el que se insertan. Partiendo de una investigación de corte cualitativo, basada en el análisis documental y de fuentes secundarias, el artículo muestra que los instrumentos curriculares que regulan la AEPI poseen intenciones reguladoras que dialogan con los mapas de provisión. Para ello, se describen primero los instrumentos curriculares con foco en su intención reguladora, es decir, el tipo de transformaciones que promueven en el mapa de la provisión. Luego, se examina de qué manera las intenciones reguladoras aspiran o no a modificar la 
unidad-diferenciación de los mapas de provisión en los que se insertan. El artículo argumenta que en mapas heterogéneos y desiguales los instrumentos sectoriales tienen altas probabilidades de mantener y reforzar las diferenciaciones existentes. En estos contextos, la elaboración de instrumentos abarcadores o cohesionadores podría favorecer una mayor justicia educativa.

Palabras clave: currículum, intención reguladora, nivel inicial, primera infancia, provisión educativa

\section{Abstract}

Early childhood care and education (ECCE) has become a priority in education policies within Latin America and across the globe. This has been paired with the progressive institutionalization of provision and the development of the curricula. However, a fragmented scenario prevails in most countries: ECCE is provided in a variety of formats and under the responsibility of different organisms and authorities. This article examines the interaction between the main curricular documents and the map of educational provision in which they are meant to regulate, in six different countries and one jurisdiction. This required a qualitative study which involved in-depth analysis of documents and secondary sources, and showed that curricular documents have diverse regulatory intents which dialogue with the maps of provision. Thus, this paper starts by describing the curricular documents and focusing on their regulatory intent, that is, the type of map transformations they promote. Secondly, it examines whether these intents aspire to modify the maps they are embedded in, in terms of unity-differentiation. The paper argues that in heterogeneous and unequal maps, there is a high probability that the documents will reinforce existing differences. In these contexts, comprehensive and cohesive documents could favor educational justice.

Key Words: curriculum, early childhood, education provision, preschool, regulatory intent

\section{Introducción}

La atención y educación de la primera infancia (AEPI) ${ }^{1}$ se ha convertido progresivamente en una prioridad para las políticas educativas en distintos países de latinoamérica y del mundo. Por un lado, a partir de la Convención sobre los Derechos del Niño se ha reafirmado que los niños y niñas son sujetos plenos de derechos, cuyo reaseguro debe ser garantizado por el Estado (Repetto, Langou, Aulicino, Achával \& Acuña, 2016). Por otro lado, un creciente cuerpo de investigaciones ha evidenciado los beneficios de la atención de la primera infancia (Irwin, Siddiqi \& Hertzman, 2007; Nores \& Barnett, 2010; Treviño et al., 2010; UNESCO, 2006). La importancia creciente de la primera infancia en la agenda política estuvo acompañada por una institucionalización cada vez mayor de las distintas modalidades de AEPI. En el campo de las políticas, esto se reflejó en el desarrollo de acciones tendientes a aumentar el acceso y la calidad en la educación de nivel inicial. En particular, en los últimos años, se han elaborado instrumentos curriculares que regulan la organización de las instituciones que se ocupan de los más pequeños (Kramer, 2016; Machado \&

$1 \mathrm{Si}$ bien pueden existir énfasis diferenciales, toda provisión educativa para la primera infancia involucra acciones de crianza, de cuidado y de enseñanza. El artículo utiliza el concepto de atención y educación de la primera infancia dada la ausencia de un concepto que permita nombrar la diversidad de formatos educativos para la primera infancia, y en tanto ha sido utilizada por diferentes organismos internacionales e investigadores para referirse a ellos. 
Lockmann, 2014; Terigi, 2002) y, en algunos casos se ha avanzado también en la elaboración de directrices curriculares orientadas a la formación de profesores y/o profesionales, en un intento por jerarquizar esta tarea (Diker, 2002).

El desafío fundamental que enfrentan los instrumentos curriculares para la primera infancia es el de regular un panorama de AEPI sumamente heterogéneo. En la mayor parte de los países $-\mathrm{y}$ a diferencia de la escuela primaria - la oferta excede a las instituciones que dependen del sistema educativo (Fromber \& Williams, 2012). Predomina, en cambio, un mapa fragmentado, compuesto por un mosaico de establecimientos muy diversos entre sí y que responden a distintas rectorías ${ }^{2}$. Esta heterogeneidad nace de una división fundacional: la separación entre el cuidado y la educación. Las instituciones abocadas a lo primero emergieron como medida de bienestar social para los padres que precisaban cuidado supervisado para sus niños pequeños. Con frecuencia, esta perspectiva de cuidado ha sido ligada por algunos autores a la doctrina de la situación irregular y al tutelaje de "menores" en situación de vulnerabilidad (Llobet, 2006). Por el otro lado, aquellas instituciones abocadas a la educación aparecieron en forma de jardín de infantes o educación preescolar, como espacio de enseñanza a través del juego y de preparación para la escuela primaria (Kaga, Bennet \& Moss, 2010).

En esta misma línea, algunos análisis curriculares previos detectaron una variedad de funciones asignadas en los distintos países a las instituciones educativas de primera infancia: la asistencial, la pedagógica, la socializadora y la preparatoria de la escolaridad primaria (Bennett, 2005; Diker, 2002). El paralelo entre estas funciones y las tradiciones identificadas en el párrafo anterior es insoslayable: mientras las funciones preparatoria y pedagógica pueden ser identificadas con la enseñanza, la socializadora pareciera derivarse de la crianza, y la asistencial del cuidado. Más aun, hay quienes sostienen que estos énfasis diferenciales respecto de la función de las instituciones educativas están también presentes en los discursos de académicos del campo y de los profesionales de primera infancia (Andrade, 2013; Broström, 2006). Como señalan Kaga et al. (2010), estas raíces históricas “encarnan visiones y entendimientos diferentes sobre los niños, los objetivos de los programas, los contenidos y los abordajes" (p. 126). Sin embargo, la división entre estas perspectivas no pareciera estar saldada y se refleja en la heterogeneidad de los abordajes existentes (Bennett, 2008; UNICEF, 2011). A su vez, son varios los autores que han alertado respecto de la falsa dicotomía que se esconde detrás de la división entre cuidado y educación (Antelo, 2005). Para el campo de la primera infancia, incluso, pareciera más adecuado referirnos a la unidad de tres acciones destinadas a los niños de esta edad: la enseñanza, el cuidado y la crianza (Broström, 2006). En este artículo, se asume que la noción de educación -concebida desde una mirada amplia- unifica las tres acciones mencionadas anteriormente. Se entiende que su separación no es solo una falsa oposición, sino también un entendimiento reducido de lo que las instituciones educativas de la primera infancia efectivamente hacen.

\footnotetext{
${ }^{2}$ La función de rectoría refiere al organismo rector o responsable de un determinado servicio o sistema. La función de rectoría no solo refiere al empoderamiento normativo, sino también al apoyo político y los recursos técnicos y presupuestarios (Repetto, Langou, Aulicino, Achával \& Acuña, 2016).
} 
La provisión de AEPI pareciera estar signada por múltiples fragmentaciones derivadas de tradiciones históricas, falsas oposiciones y solapamientos. En los países latinoamericanos, dicha fragmentación se refuerza aún más al contemplar las desigualdades sociales. En este contexto, es clave analizar los instrumentos curriculares para la primera infancia a la luz de la configuración del mapa que aspiran regular. Si bien esta interacción es importante para todo estudio de políticas educativas preocupado por la justicia social (Cox, 1986), pareciera ser especialmente relevante $-\mathrm{y}$ a pesar de ello poco explorado- en el campo de la primera infancia.

En este trabajo se explora la interacción de los principales documentos curriculares de seis países y una jurisdicción con el mapa de AEPI en el que se insertan. Para ello, se caracteriza la organización de la provisión según las coordenadas de unidad-diferenciación, entendidas como un continuo. Al hacerlo, se propone complejizar análisis que prevalece en la literatura especializada que suele concentrarse en la función rectora. En segundo lugar, se describen los instrumentos curriculares según su intención reguladora, es decir, en relación al tipo de transformaciones que promueven. Luego, se muestra de qué manera la intención reguladora aspira a modificar o no las tendencias hacia la unidad-diferenciación del mapa en el que se inserta. El artículo concluye con una invitación a continuar el análisis iniciado con el foco en los países latinoamericanos y la justicia educativa.

\section{Coordenadas teórico-metodológicas}

El trabajo propone un análisis comparado del currículum prescripto, es decir, el que se encuentra plasmado en los documentos oficiales. En tanto regulación escrita, entendemos que cumple tres funciones principales (Terigi, 1999). En primer lugar, se trata de un dispositivo de la política pública que establece el tipo de experiencias educativas que se espera brindar a los alumnos. En segundo lugar, comprende una expresión de los compromisos que el Estado asume con la sociedad en general y con el sistema en particular. Por último, es una herramienta de trabajo para los equipos docentes y directivos de las instituciones educativas. Este trabajo se concentra en la segunda función, en tanto permite analizar el currículum prescripto como un instrumento vinculado a la gobernabilidad del sistema educativo (Gvirtz, 2002) -aunque en el caso de la primera infancia, tal como se anticipó, se argumenta que la gobernabilidad debe exceder al sistema educativo y alcanzar a todos los formatos institucionales que adopta la AEPI para los más chicos-.

Partiendo de esta definición, la presente indagación ${ }^{3}$ se ocupa de los principales instrumentos curriculares de Argentina, Ontario (Canadá), Chile, Escocia, Nueva Zelanda, Singapur y Suecia. Los casos fueron seleccionados a partir de los siguientes criterios: (1) disponibilidad y factibilidad para el análisis -que contaran con una base curricular común para la educación de la primera infancia disponible en

3 El presente artículo se desprende de una investigación financiada por la Fundación María Cecilia Souto Vidigal, cuyo foco estaba puesto en los procesos de desarrollo curricular en América Latina. 
línea y publicada en portugués, español, inglés o francés- (2) coherencia, claridad y rigurosidad de la base curricular - que los instrumentos representen, en relación a las dimensiones de análisis, aportes para el proceso de construcción de nuevos instrumentos curriculares en países de la región- y (3) representatividad de distintas tradiciones curriculares y presencia latinoamericana -que los casos dieran cuenta de distintas tradiciones curriculares y distintos modos de resolver la política curricular a nivel global, en general, y en América Latina, en particular.

Si en algunos países el currículum se plasma en un único documento que regula la totalidad del mapa de provisión educativa de la primera infancia, en la mayoría la normativa se encuentra constituida por una batería de documentos que, en diversos grados, con distintos formatos y alcances, se articulan para regular el trabajo de las instituciones de atención de esta franja etaria. Un estudio en profundidad del mapa curricular de cada país excedería las posibilidades de este trabajo ya que requeriría un análisis de la totalidad de las normativas vigentes.

Se optó aquí por seleccionar el documento que, en su contexto específico y en relación al resto de la normativa, tuviera un papel preponderante. Así, este estudio se enfoca en los siguientes documentos: Núcleos de Aprendizajes Prioritarios para el nivel inicial (Argentina, 2004), Early Learning for Every Child Today. A framework for Ontario early childhood setting (Ontario, 2007), Bases curriculares para la educación parvularia (Chile, 2001), The Early Years Framework (Escocia, 2008), Te Whàriki (Nueva Zelanda, 1996), Nurturing Early Learners: a curriculum framework for Kindergartens in Singapore (Singapur, 2012) y Curriculum for the Preschool Lpfö 98 (Suecia, 2010). Se consideraron documentos vigentes que hubieran sido desarrollados en los últimos veinte años, en tanto responden a la preocupación por el acceso y la calidad en la educación de nivel inicial mencionada.

El análisis interpretativo de los documentos fue objeto de una aproximación cualitativa. La lectura comparada y reiterada de los instrumentos curriculares se valió de la codificación abierta, axial y selectiva. Como fue señalado, este trabajo se centra en el análisis de los textos curriculares y no pretende aventurar conclusiones relacionadas con los efectos de su implementación. El concepto de intención reguladora permitió focalizar la interpretación en los propósitos manifiestos en cada uno de los documentos. Para lograr una comprensión más acabada de estos objetivos se hizo evidente, además, la necesidad de recurrir a un análisis de lo que se denominó "mapas de AEPI", es decir, de la configuración de la provisión en cada país. El argumento central del presente artículo se desprende del análisis de los instrumentos curriculares en relación a los mapas de AEPI de cada país o jurisdicción. Para ello, al análisis documental se sumó el relevamiento de fuentes secundarias que permitieron caracterizar los mapas de provisión de cada país. Se utilizaron los sitios web de las distintas dependencias de gobierno involucradas en la atención de la primera infancia, consultas a informantes claves y documentos de organismos internacionales o nacionales que ofrecieran análisis pertinentes. 


\subsection{Los mapas de AEPI}

La heterogeneidad de los mapas de AEPI obliga a extender la mirada más allá del sistema educativo y la educación inicial. En muchos países, la AEPI toma formatos institucionales diversos y se encuentra bajo distintas rectorías, y se caracteriza por tener distintas lógicas y propósitos. Las raíces de la mencionada heterogeneidad son históricas y se retraen a los orígenes de las políticas de atención de la primera infancia: mientras las instituciones de "cuidado" se ocuparon de niños en situación de vulnerabilidad desde un enfoque "asistencial", las instituciones "educativas" se ocuparon del resto, adoptando un propósito "educativo". En el plano de la organización política, esta diversidad se manifiesta en los diferentes tipos de oferta y funciones de rectoría: mientras los establecimientos de "cuidado" suelen depender de los organismos responsables de la protección social y están orientados a los sectores vulnerables, las instituciones "educativas" suelen depender del sector educativo y estar dirigidos a los sectores medios y acomodados (Moss, 2007). Estas diferencias han marcado a fuego la situación de la regulación, provisión y financiamiento de la atención de la primera infancia (Bennett, 2008).

Sumado a lo anterior, hay que contemplar la tardía consolidación de la educación inicial como nivel educativo y la aún más reciente institucionalización de la AEPI orientada a los primeros tres años de vida. De hecho, mientras algunos organismos internacionales -UNESCO, por ejemplo- la delimitan entre los cero y los ocho años, otros focalizan su definición entre los cero y los tres o cuatro años ${ }^{4}$. En línea con lo observado anteriormente, se suele ofrecer servicios de "cuidado" a los niños más pequeños -habitualmente de cero a dos años- y de "educación" a los niños más grandes - usualmente de tres a cinco años.

Asimismo, a diferencia de otros niveles educativos, la AEPI no surgió con la intención de ser universal ni homogeneizadora (Bloch, Cannella \& Swandener, 2014; Cannella, 1997). Prueba de ello es que las discusiones respecto de la obligatoriedad de la educación inicial sean materia de la agenda actual. Todas estas cuestiones contribuyen a que, en la mayoría de los países, la unificación del mapa de AEPI devenga de una serie de esfuerzos de la política pública por amalgamar un conjunto de servicios históricamente diferenciados.

La literatura sobre la organización de la AEPI se ha centrado en la identificación y análisis de dos tendencias: la fragmentación y la integración (Bennett, 2003, 2008). Mientras la primera refiere a un mapa desmembrado y heterogéneo, la segunda caracteriza a mapas cuya provisión es uniforme, se encuentra amalgamada o está fuertemente articulada. Estas tendencias han sido principalmente estudiadas respecto de la función de rectoría, que hace referencia al

4 La definición de la franja etaria está atravesada por tradiciones históricas, discursos expertos informados por los saberes de la medicina y la psicología del desarrollo, principalmente-, prácticas político-culturales y umbrales prexistentes, tales como la edad de inicio de la escuela primaria y por la existencia y la duración de las licencias por maternidad y/o paternidad. Este artículo analiza la AEPI desde el momento en el que finalizan las licencias por maternidad y/o paternidad y hasta el inicio de la escolaridad primaria en cada país y jurisdicción estudiada. 
organismo a cargo del servicio (Bennett, 2008; Kaga et al., 2010; Repetto et al., 2016).

A pesar de la importancia de esta discusión, se sugiere aquí que presenta algunas limitaciones para dar cuenta de la complejidad de los mapas de AEPI. Este artículo avanza en el estudio del mapa en función de las tendencias hacia la unidad o diferenciación (Braslavsky, 1982) ${ }^{5}$ del formato de AEPI y en relación a dos ejes. El eje vertical se corresponde con la variable edad -desde cuándo y hasta cuándo, así como con qué ciclado se delimita la provisión educativa orientada a la primera infancia- y el horizontal con la variable grupos sociales -qué formatos de AEPI se ofrecen a distintos grupos sociales-. Así, si todos los grupos sociales y edades reciben una provisión uniforme se habla de una tendencia a la unificación. En cambio, cuando hay una distinción, ya sea por grupos de edad o grupos sociales, es posible visualizar una tendencia a la diferenciación. En el Gráfico 1, cada unidad de análisis ha sido posicionada según el grado de unificación o diferenciación. Dicha posición ha sido determinada con criterios analíticos cualitativos y representa una configuración del mapa de provisión de AEPI. En el Cuadrante I, la provisión es unificada tanto vertical como horizontalmente, es decir, se provee de modo uniforme a los niños de todas las edades y todos los grupos sociales. En el Cuadrante II, la provisión es unificada en relación a la edad, pero es diferenciada para los distintos grupos sociales. En el Cuadrante III, la heterogeneidad es máxima: hay diferenciación tanto en la provisión de AEPI por edad y por grupo social. Por último, en el Cuadrante IV, la provisión según la edad es diferenciada, pero es uniforme para todos los grupos sociales.

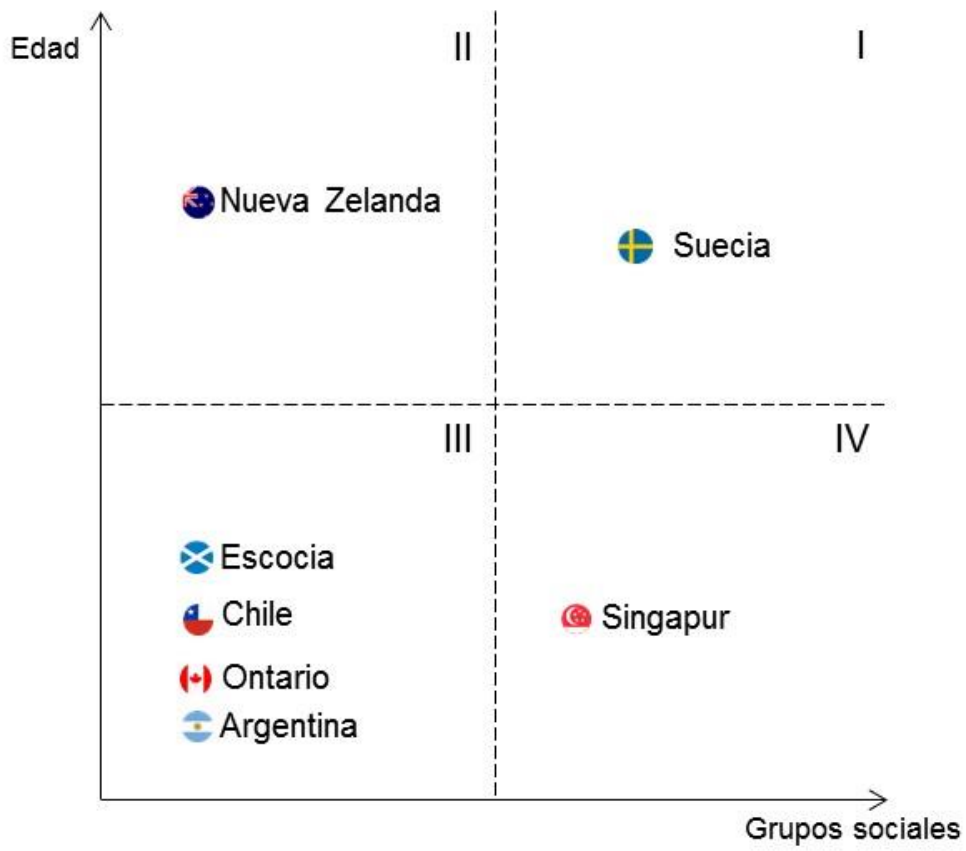

Gráfico 1. La unidad-diferenciación de la provisión de AEPI por edad y grupos sociales

Fuente: elaboración propia.

5 Optamos por recuperar los conceptos de unidad y diferenciación (Braslavsky, 1982), ya que parecen describir de manera más precisa las tendencias analizadas sin asignarles carga valorativa a priori. 
Entre los mapas analizados, el más unificado es el de Suecia, cuya Förskola ${ }^{6}$ (educación preescolar) es una unidad pedagógica que atiende a los niños de entre uno y cinco años con una cobertura del $95 \%$. El proceso de unificación del mapa ${ }^{7}$ comenzó en la década de 1970 con la unificación de la oferta. En ese entonces, Suecia integró la oferta ligada al "cuidado" y a la "educación" y reorganizó su provisión en instituciones preescolares con una fuerte orientación hacia el juego, la exploración y la creatividad (Lohmander \& Samuelsson, 2015). Más tarde, en la década de 1990 el proceso culminó con la unificación rectora en el sector educativo (Taguma, Litjens, Makowiecki \& Early, 2013).

Nueva Zelanda, en cambio, ofrece una provisión diferenciada por grupos sociales, con el objetivo de respetar la biculturalidad que caracteriza al país ${ }^{8}$. Así, los formatos son variados e incluyen programas liderados por docentes (teacher-led) y por las comunidades (parent-led). Entre los primeros, se encuentran los jardines de infantes que atienden a los niños de entre dos y cinco años en los que la totalidad de los docentes se encuentran certificados, mientras que entre los segundos se encuentran los Te Kōhanga Reo, una modalidad de inmersión total en la cultura maorí desde el nacimiento hasta los cinco años llevado adelante por miembros de esta comunidad.

En contraste, Chile ${ }^{9}$ tiene una provisión diferenciada: la provisión de AEPI y la fiscalización de la calidad se dan en una multiplicidad de formatos institucionales y bajo regulaciones diversas (Morales, 2013). La diferenciación por edad está dada porque los principales proveedores públicos en el tramo de cero-tres años son la Junta Nacional de Jardines Infantiles y Fundación INTEGRA. Mientras tanto, son los municipios quienes proveen educación parvularia en la franja de cuatro y cinco años. Esta última franja se encuentra mayormente incorporada a escuelas primarias, y es por ello que su provisión depende del Ministerio de Educación ${ }^{10}$.

Por su parte, el caso de Singapur es diferenciado por edad en los términos que plantea Bennett (2008), en tanto el Estado sólo se hace responsable de la oferta de

$6 \mathrm{El}$ año previo al inicio de la escolaridad obligatoria, el sistema escolar sueco posee un año denominado Förskoleklass que combina los métodos y contenidos propios del nivel inicial con los del nivel primario.

7 Para conocer más sobre el proceso de unificación del mapa de provisión en Suecia puede leerse Kaga et al. (2010).

8 Podmore (2002) ofrece más información sobre la provisión educativa para la primera infancia en Nueva Zelanda y Lourie (2016) presenta un análisis detallado de las políticas educativas orientadas a la biculturalidad en Nueva Zelanda. Asimismo, Kaga et al. (2010) describe el proceso de unificación del mapa de provisión educativa en Nueva Zelanda

9 Cabe destacar que en el caso de Chile el proceso de reforma, aunque liderado por el MINEDUC, incluyó la consulta a un número importante de actores ligados a la atención de la primera infancia. Para un relato detallado sobre este proceso pueden leerse los trabajos de Peralta, Arancibia Lorca \&Blanco Guijarro (2004).

10 El caso de Chile invita a un análisis más detallado que incorpore el tipo de gestión de las instituciones de primera infancia y la segmentación de la provisión respecto del nivel socioeconómico. Debe notarse que en el sector privado o particular encontramos proveedores para el total de la franja cero-cinco años con o sin subvención municipal. En futuros trabajos se espera poder avanzar en esta dirección, más allá de las breves consideraciones que se exponen en las conclusiones. 
servicios para una franja etaria - habitualmente de los tres o cuatro años en adelante- y relega en las familias la atención de los niños más pequeños. El subsistema de educación preescolar en Singapur se extiende de los tres a los seis años. Allí, el Estado no regula la franja de 18 meses a 3 años, que es responsabilidad de las familias -aunque según sus ingresos pueden obtener subsidios ${ }^{11}$.

Uno de los mapas que presenta una mayor diferenciación es el argentino. En ese país, la educación inicial -que se extiende entre los cuarenta y cinco días y los cinco años- depende del Ministerio de Educación y constituye una unidad pedagógica compuesta de dos ciclos: el jardín maternal -cuarenta y cinco días a dos años- y el jardín de infantes - tres a cinco años-. No obstante, el nivel inicial atiende solo a una porción de los niños y niñas -especialmente en el ciclo maternal-, mientras que el resto de la provisión está en manos de instituciones dependientes del Ministerio de Desarrollo Social - los Espacios de Primera Infancia- y una variedad de formatos no integrados en el sistema educativo, regulados solo parcialmente por el Estado ${ }^{12}$. Algunos de ellos son de base comunitaria o gremial y otros dependen orgánica y funcionalmente de los niveles jurisdiccionales y municipales de todo el país (Rozengardt, 2014) ${ }^{13}$.

\subsection{Los instrumentos curriculares}

Los instrumentos curriculares seleccionados se diferencian, en principio, según el grado y tipo de concreción curricular. Haciendo a un lado esta primera diferencia, los instrumentos curriculares se analizaron en torno a su intención reguladora. Esta noción se refiere al tipo y escala de las transformaciones que el instrumento curricular busca promover en el mapa en el que se inserta. Los instrumentos se orientan diferencialmente a modificar los límites verticales $\mathrm{y} / \mathrm{u}$ horizontales del mapa (Cox, 1986), es decir, pueden tener como intención modificar o abstenerse de operar sobre las tendencias del mapa, sean estas de diferenciación o integración.

En relación a esta variable central se identificaron dos grandes grupos de instrumentos. Por un lado, están los que proponen regular exclusivamente a las instituciones del sistema escolar -es decir, a la educación inicial-, a los que denominamos sectoriales. Estos son los casos de los instrumentos curriculares de Argentina, Chile, Singapur y Suecia, los cuales están ligados a y se desprenden del sistema educativo.

Por otro lado, entre los instrumentos que posan su mirada sobre el mapa en su conjunto y procuran englobar todos los formatos de AEPI, distinguimos entre instrumentos abarcadores y cohesionadores según su intención reguladora. Los

11 Más información sobre la provisión de educación infantil en Singapur puede leerse en Ebbeck \& Chan (2011) y en Ting (2007).

12 Más información sobre las instituciones no incorporadas al sistema educativo oficial puede encontrarse en Zibecchi (2013).

13 Para conocer más sobre la fragmentación que prima en mapa de la provisión argentino se sugiere leer Repetto et al. (2016), Rozengardt (2014) y Redondo (2012). 
primeros, como el de Nueva Zelanda, pretenden regular a todas las modalidades en un mismo documento sosteniendo su especificidad. Aunque presentes y reguladas por el mismo currículum, los distintos formatos son reconocidos y sostenidos en sus diferencias, tal como queda en evidencia en el siguiente fragmento del instrumento curricular neozelandés:

"El currículum de la primera infancia ha sido concebido como un whàriki, o tejido/alfombra, tejido desde sus principios, hilos y objetivos definidos en este documento. El concepto de whàriki reconoce la diversidad de formas que toma la educación de la primera infancia en Nueva Zelanda. Diferentes programas, filosofías, estructuras y ambientes contribuirán a los distintos patrones del tejido" (Te Whàriki, 1996, p. 11).

Por su parte, los instrumentos cohesionadores buscan enlazar o amalgamar los formatos existentes -estos son los casos de Ontario y Escocia-. En ambos instrumentos, la cohesión a la que se aspira está motivada por el intento de superar la separación entre cuidado y educación. Así lo expresa el documento Early Learning for Every Child Today. A framework for Ontario early childhood settings: "cuidado y educación son conceptos inseparables, (...) todos los servicios de calidad proveen tanto cuidado como educación" (p. 4). De alguna manera, se puede afirmar que ambos casos utilizan la regulación curricular para avanzar hacia la unificación de su mapa, tal como lo afirma el marco curricular escocés:

“El paisaje de los servicios para la primera infancia está bastante fragmentado en términos de la provisión del servicio y de la fuerza de trabajo (...). Un cambio profundo es necesario para reconceptualizar las influencias en los niños y los primeros años, para verlos como partes complementarias de un sistema global alrededor del niño" (The Early Years Framework, p. 15)

En este sentido, los instrumentos cohesionadores asumen una tarea desafiante en el marco de un proceso de transformaciones que los exceden.

En síntesis, se identifican dos grupos de documentos -los que se atienen al sistema educativo y aquellos que pretenden regular todo el mapa de AEPI- y se distinguen tres tipos de intenciones reguladoras que pueden tener -sectorial en el caso del primer grupo, y abarcadora o cohesionadora en el segundo. Caracterizar en mayor profundidad cada uno de los instrumentos curriculares aquí analizados es fundamental en tanto cada tipo de intención reguladora se deja entrever no sólo en el texto del documento, sino también en la institución autora, sus destinatarios, sus características, la franja etaria a la cual está dirigida, y las competencias de los responsables de su armado. En la Tabla 1, es evidente que cada una de estas características se alinea con la intención reguladora del instrumento curricular. 
Tabla 1

Características de los instrumentos curriculares de Argentina, Ontario (Canadá), Chile, Escocia, Nueva Zelanda, Singapur y Suecia

\begin{tabular}{|c|c|c|c|c|c|c|c|}
\hline & Argentina & $\begin{array}{l}\text { Ontario } \\
\text { (Canadá) }\end{array}$ & Chile & Escocia & $\begin{array}{l}\text { Nueva } \\
\text { Zelanda }\end{array}$ & Singapur & Suecia \\
\hline $\begin{array}{l}\text { Intención } \\
\text { reguladora }\end{array}$ & Sectorial & $\begin{array}{l}\text { Cohesiona } \\
\text {-dora }\end{array}$ & Sectorial & $\begin{array}{l}\text { Cohesionad } \\
\text {-ora }\end{array}$ & Abarcadora & Sectorial & Sectorial \\
\hline $\begin{array}{l}\text { Franja } \\
\text { etaria }\end{array}$ & $\begin{array}{l}45 \text { días- } 6 \\
\text { años }\end{array}$ & 0-6 años & $\begin{array}{l}85 \text { días- } 6 \\
\text { años }\end{array}$ & $0^{14}-8$ años & 0-6 años & 3-6 años & 1-5 años \\
\hline $\begin{array}{l}\text { Elaboració } \\
\mathrm{n}\end{array}$ & $\begin{array}{l}\text { Equipo } \\
\text { técnico } \\
\text { curricular }\end{array}$ & $\begin{array}{l}\text { Panel de } \\
\text { expertos }\end{array}$ & $\begin{array}{l}\text { Equipo } \\
\text { técnico } \\
\text { curricular }\end{array}$ & $\begin{array}{l}\text { Panel de } \\
\text { expertos y } \\
\text { COSLA }\end{array}$ & $\begin{array}{l}\text { Representante } \\
\text { s de las } \\
\text { distintas } \\
\text { modalidades }\end{array}$ & $\begin{array}{l}\text { Equipo } \\
\text { técnico } \\
\text { curricular }\end{array}$ & $\begin{array}{l}\text { No hay } \\
\text { informa- } \\
\text { ción }\end{array}$ \\
\hline $\begin{array}{l}\text { Proceso de } \\
\text { elabo- } \\
\text { ración }\end{array}$ & Tecnocrático & Experto & $\begin{array}{l}\text { Tecnocrá- } \\
\text { tico }\end{array}$ & Experto & Deliberativo & $\begin{array}{l}\text { Tecnocrá- } \\
\text { tico }\end{array}$ & $\begin{array}{l}\text { No hay } \\
\text { informa- } \\
\text { ción }\end{array}$ \\
\hline $\begin{array}{l}\text { Destinata- } \\
\text { rios }\end{array}$ & $\begin{array}{l}\text { Docentes de } \\
\text { educación } \\
\text { inicial y } \\
\text { equipos } \\
\text { curriculares } \\
\text { jurisdiccional } \\
\text { es }\end{array}$ & $\begin{array}{l}\text { Adultos } \\
\text { que } \\
\text { trabajan } \\
\text { en las } \\
\text { distintas } \\
\text { modalidad } \\
\text { es }\end{array}$ & $\begin{array}{l}\text { Docentes } \\
\text { de } \\
\text { educación } \\
\text { parvularia }\end{array}$ & $\begin{array}{l}\text { Gobierno } \\
\text { escocés, } \\
\text { aliados } \\
\text { locales y } \\
\text { adultos que } \\
\text { trabajan en } \\
\text { las distintas } \\
\text { modalida- } \\
\text { des }\end{array}$ & $\begin{array}{l}\text { Adultos que } \\
\text { trabajan en } \\
\text { las distintas } \\
\text { modalidades }\end{array}$ & $\begin{array}{l}\text { Profesio- } \\
\text { nales que } \\
\text { trabajan } \\
\text { en el pre- } \\
\text { escolar }\end{array}$ & $\begin{array}{l}\text { Adultos que } \\
\text { trabajan en } \\
\text { el } \\
\text { preescolar }\end{array}$ \\
\hline
\end{tabular}

Fuente: elaboración propia.

Se tomarán tres casos - cada uno con una intención reguladora diferente -para dar cuenta de la relación que existe entre estas características. En primer lugar, en el caso de Singapur, el instrumento curricular se ubica entre los que cuentan con una intencionalidad sectorial y, dado que se ocupa del sistema educativo, se orienta a la franja etaria que comprende a los preescolares (de tres a seis años). El documento es producido por el Ministerio de Educación, organismo que detenta la función rectora del nivel preescolar, y su elaboración fue llevada adelante por el equipo técnico curricular de dicho ministerio. Tanto en este como en los documentos sectoriales analizados predomina un proceso de elaboración tecnocrático, que es el estilo de elaboración habitual de los instrumentos curriculares en el sistema educativo (Haft \& Hopmann, 1990). Por otra parte, el instrumento singapurense está destinado a los profesionales que trabajan en los preescolares. La referencia amplia a profesionales está vinculada con una variedad de certificaciones con distintos grados de

14 Dado que Escocia se propone incluir a los servicios de atención prenatal en su mapa, formalmente establecen que el marco regula desde el momento de la concepción. 
responsabilidad, tareas y tipos de instituciones ${ }^{15}$. Así, otra característica de los instrumentos curriculares sectoriales es que sus destinatarios son los profesionales que se desempeñan en las instituciones de educación inicial, es decir, actores del sistema educativo. Por último, se trata de un diseño curricular que presenta la selección, organización y secuenciación de los contenidos a enseñar. Asimismo, hace referencia a las estrategias de enseñanza, a la planificación y a la evaluación de los aprendizajes. En este sentido, se trata de un documento característico del sector educativo, que puede encontrarse también en otros niveles, y que reafirma la intencionalidad de regular exclusivamente dicho sistema.

Por su parte, el caso de Nueva Zelanda, cuyo documento es de tipo abarcador, incluye a los niños desde el nacimiento y hasta el inicio de la escolaridad primaria, dado que algunas de las modalidades educativas que se encuentran bajo su cobijo específicamente las maoríes -son comprehensivas y atienden a los niños desde su nacimiento. Además, la autoría del Ministerio de Educación se explica porque, al momento de su elaboración, este país ya había transferido la rectoría de todos los formatos de AEPI a dicho Ministerio. Su elaboración fue liderada por representantes de las distintas modalidades de AEPI y su desarrollo fue de tipo deliberativo. Tal como se expresa en el documento ECEC curriculum of New Zealand, TeWhàriki (Ministry of Education, 1996):

"mientras que los servicios son diversos en términos de estructuras y filosofías, el personal de los servicios de primera infancia trabajó en conjunto para desarrollar un currículum común en el que basar sus programas. Fue desarrollado a partir de y construido en base a la experiencia de desarrollo curricular los servicios curriculares, en conjunto con los hallazgos de investigación, la literatura internacional y el conocimiento compartido y los consensos construidos que emergieron en Nueva Zelanda a lo largo de las últimas dos décadas" (p. 8)

Sus destinatarios se definen de un modo más amplio que en un documento de tipo sectorial. En particular, esto se debe a que, como fue señalado anteriormente, la modalidad maorí no es atendida por docentes certificados sino por miembros de la comunidad. Así, el desarrollo deliberativo del documento y la referencia a adultos que trabajan en los distintos formatos de AEPI como destinatarios resulta consistente con la intención reguladora de tipo abarcador. Finalmente, se trata de un documento que se encuentra a medio camino entre un marco o guía, y un diseño curricular ya que tiene la intención de regular a todas las modalidades en un mismo documento sosteniendo su especificidad: mientras la base aspira a brindar especificaciones para la enseñanza, la variedad de formatos a la que se orienta supone cierta apertura y flexibilidad.

15 Las cualificaciones difieren según las posiciones a ocupar en las distintas instituciones para la primera infancia. El complejo sistema de certificaciones Singapore Workforce Skills Qualifications System for Early Childhood Care \& Education consiste en la definición de competencias validadas por empleadores y cada certificación supone la posesión de un conjunto específico de competencias. Más información puede encontrarse Ting (2007). 
Por último, el instrumento escocés ${ }^{16}$ tiene una intención de tipo cohesionador. Dado que el Early Years Framework aborda todos los servicios orientados a la atención de la primera infancia, su alcance incluye a los servicios de salud, a los niños desde su concepción y se extiende más allá del inicio de la escolaridad primaria, hasta los ocho años. Así, el instrumento escocés se enmarca en un conjunto más amplio de esfuerzos orientados a cohesionar el mapa de la provisión. Por la misma razón, se contrata a un panel de expertos que analiza el mapa de la provisión existente ${ }^{17}$ y elabora el instrumento curricular que, al ser un plan de acción que establece objetivos a corto, mediano y largo plazo para cohesionar el mapa de provisión educativa, requiere de la firma y el consenso de la Convención de Autoridades Escocesas Locales y el gobierno escocés, a modo de compromiso entre las partes. Lo anterior nos remite a la función del currículum como expresión de los compromisos del Estado con la sociedad. También resulta congruente con la intención reguladora que el gobierno escocés, aliados locales y adultos que trabajan en los distintos formatos de AEPI sean los destinatarios del documento. Aunque se ofrecen algunas orientaciones generales, como la valoración del juego, los contenidos se encuentran en los documentos curriculares complementarios.

\section{Los instrumentos curriculares en sus mapas de provisión}

La intención reguladora funciona como variable bisagra para examinar el modo en el que los instrumentos curriculares dialogan con los mapas de provisión en los que se insertan. En este apartado se examina el modo en que los distintos tipos de intenciones reguladoras operan en los distintos mapas de provisión. En otras palabras, una misma intención puede tener distintos efectos según las características del mapa en el que se inserte. Se procura identificar aquí aquellas interacciones en las cuales la intención reguladora favorezca la unificación en algunos de los dos ejes mencionados. Cabe remarcar que los movimientos que se describen a continuación no son efectivos, sino potenciales, esto es, se derivan únicamente de la intención reguladora del documento y no de un análisis de los cambios efectivos acontecidos en cada país o jurisdicción.

En el Gráfico 2 se plasman dos componentes que permitirán apreciar las conclusiones del presente estudio en torno esta interacción: cada uno de los mapas de AEPI descriptos aparece posicionado según su unificación vertical y horizontal y las flechas azules indican la tendencia que favorece la intención reguladora del instrumento curricular analizado.

16 Más información sobre la provisión de educación infantil en Escocia puede leerse en Austin (2013) y Clark \& Waller (2007).

17 Los resultados de su trabajo constituyen la Parte 2 de la base curricular, que funciona a modo de justificación de las decisiones tomadas. 


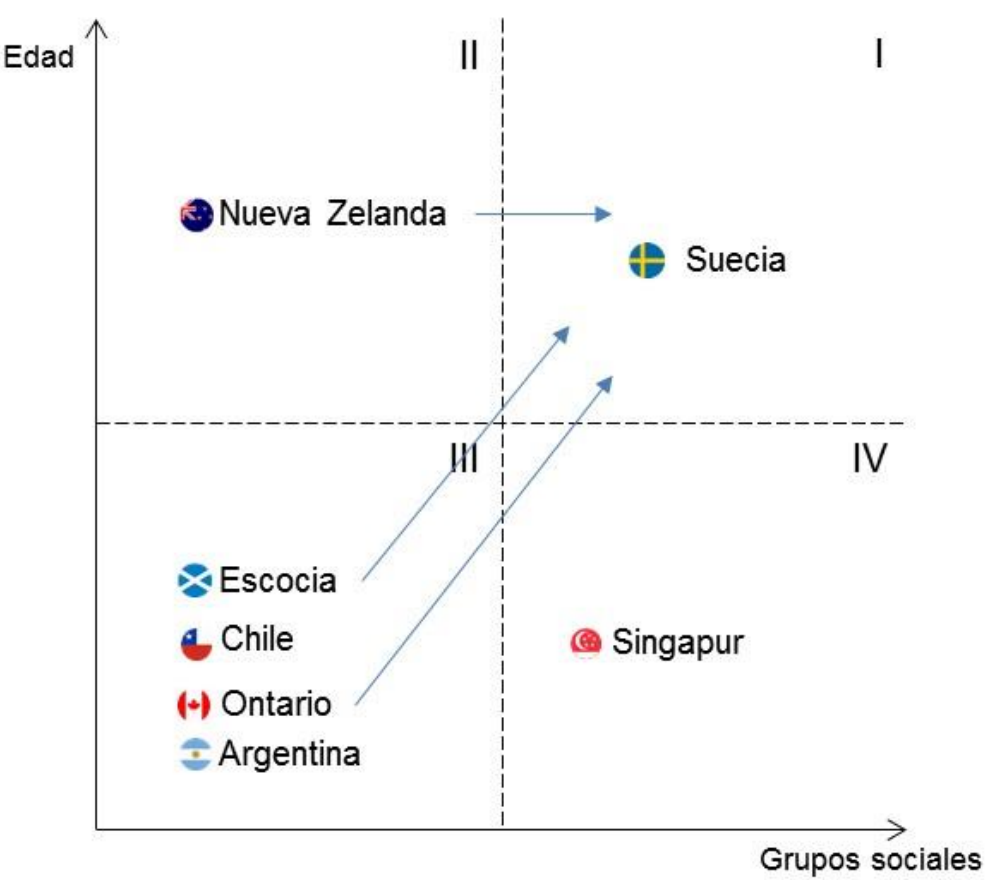

Gráfico 2. La intención reguladora de los instrumentos curriculares en función del mapa de provisión en el que se insertan

Fuente: elaboración propia.

A partir de la descripción de los mapas, del análisis de los documentos curriculares y su intención reguladora, y de la interacción entre ambos se pueden apreciar algunos patrones. En primer lugar, los documentos de intencionalidad sectorial no aspiran a unificar el mapa de atención. En este sentido, Argentina, Chile, Singapur y Suecia conservan sus posiciones en términos de su unificación vertical y horizontal en tanto los documentos sectores no aspiran a unificar el mapa. Mientras tanto, el resto de los instrumentos aspira a unificar los mapas de AEPI en alguna dirección. En el caso de Nueva Zelanda, la unificación se deriva de la articulación de la oferta de AEPI bajo una misma regulación. A pesar de que se respetan las diferencias entre formatos, su articulación apunta a una mayor unificación horizontal - lo que se grafica en el movimiento de Nueva Zelanda desde el cuadrante II hacia el cuadrante I-. En cuanto a Escocia y Ontario, el carácter cohesionador de la regulación aspira a unificar el mapa otorgándole una mayor cohesión -movimiento que puede observarse en el movimiento del cuadrante III al I-.

De esta manera, los efectos potenciales de los instrumentos curriculares para la primera infancia están delimitados por la interacción de la intención reguladora de cada documento con el mapa de la provisión de cada caso de estudio. En términos de Cox (1986), puede argumentarse que los instrumentos abarcadores y los cohesionadores aspiran a modificar los límites horizontales $-\mathrm{y}$ como consecuencia, verticales- del mapa de la atención. Mientras tanto, los instrumentos sectoriales mantienen el status quo. 
Al explorar las implicancias de las intenciones reguladoras del currículum, es evidente que las tendencias a la unificación y a la diferenciación adquieren relevancia cuando se las pone bajo la lupa de la justicia educativa y se las interroga mediante consideraciones de redistribución y reconocimiento (Veleda, Rivas \& Mezzadra, 2011).

Tanto aquellos países con mapas de AEPI diferenciados, tales como Argentina y Chile, como aquellos otros que se encuentran en la vereda contraria, como Suecia, poseen instrumentos curriculares sectoriales. Sin embargo, un instrumento sectorial inserto en un mapa unificado como el sueco tiene un alcance mayor que un instrumento similar en un mapa diferenciado como el chileno o el argentino. En este último caso, el carácter heterogéneo de las instituciones que atienden a esta franja etaria convive con una regulación sectorial (y homogénea) que puede resultar inadecuada y poco eficaz (Diker, 2002) considerando que la educación inicial o infantil alcanza únicamente a una porción de los niños. Mientras tanto, la regulación sectorial no alcanza a otros formatos institucionales de AEPI que no están integrados al sistema educativo oficial y que suelen recibir a los niños de sectores socioeconómicos más bajos. En este sentido, la regulación del sector podría contribuir a mantener e incluso reforzar las injusticias educativas existentes.

Por su parte, el instrumento neozelandés pareciera favorecer el reconocimiento de las diferencias culturales. Su estudio hace evidente de qué manera las diferentes realidades políticas, sociales, culturales y económicas condicionan y atraviesan los servicios de atención de la primera infancia. En particular, muestra cómo un aspecto social clave como la biculturalidad puede ser incorporado en la elaboración, en la organización y en el enfoque de una base curricular para regular la atención de la primera infancia con un criterio de justicia orientada al reconocimiento.

Finalmente, en el caso de los instrumentos cohesionadores la tendencia hacia la unificación podría tener efectos redistributivos en mapas diferenciados, especialmente en ocasiones en las que la diferenciación se encuentra anclada en desigualdades sociales y en las que los sectores acomodados acceden a más y mejor educación que sus pares menos aventajados. La unificación del mapa mediante un instrumento cohesionador podría favorecer que el documento alcanzara a toda la primera infancia.

\section{Conclusiones}

El artículo examinó los principales instrumentos curriculares para la primera infancia de Argentina, Chile, Escocia, Nueva Zelanda, Ontario (Canadá), Singapur y Suecia. Dado el desafío que representa regular panoramas de AEPI sumamente heterogéneos, dicho análisis fue contextualizado, es decir, teniendo en cuenta las características del mapa de cada país o jurisdicción. Se utilizó la intención reguladora como bisagra para poner en diálogo a estos instrumentos con las 
características de unidad-diferenciación de los mapas de AEPI por edad y grupos sociales.

De lo anterior se desprende que una misma intención puede tener distintos efectos potenciales según las características del mapa en el que se inserte. En mapas heterogéneos y desiguales los instrumentos sectoriales tienden a mantener y reforzar las desigualdades existentes en la sociedad. Este pareciera ser el caso de muchos países latinoamericanos, en los que la provisión se diferencia por sectores socioeconómicos que reciben AEPI de desigual calidad. En estos contextos, la elaboración de instrumentos abarcadores o cohesionadores podría favorecer una mayor justicia educativa en materia de AEPI.

Al dar un paso más, podría considerarse que el análisis de los instrumentos curriculares es una puerta de entrada a una discusión más amplia. Para ello, es necesario que quienes se ocupan de pensar y actuar sobre la AEPI adopten una mirada que reconozca la totalidad de formatos institucionales que la componen. Se trata de una perspectiva que considere los efectos de la heterogeneidad del mapa sobre las desigualdades sociales en los más pequeños y formule políticas que tiendan a reducir la brecha socioeconómica. Así, el artículo deja abierta la invitación a otras indagaciones que permitan avanzar en la construcción de una mayor justicia educativa para la primera infancia en América Latina.

\section{Referencias bibliográficas}

Andrade, D. (2013). A criança nas entrelinhas dos discursos de acadêmicos brasileiros sobre professor da educação infantil. Revista de Educação Pública, 22(49/1), 361-377.

Antelo, E. (2005). La falsa antinomia enseñar-asistir. Diario La Capital, Rosario, publicación del 11-06-05.

Austin, G.R. (2013). Early childhood education: An international perspective. London: Academic Press.

Bennett, J. (2003). The persistent division between care and education. Journal of early childhood research, 1(1), 21-48.

Bennett, J. (2005). Curriculum issues in national policy-making. European early childhood education research journal, 13(2), 5-23.

Bennett, J. (2008). Early childhood education and care systems in the OECD countries: the issue of tradition and governance. Encyclopedia on Early Childhood Development, 1-5.

Bloch, M., Cannella, G.S., \& Swadener, B.B. (2014). Reconceptualizing Early Childhood Care and Education: Critical Questions, New Imaginaries and Social Activism. New York: Peter Lang. 
Braslavsky, C. (1982). Conceptos centrales de política educativa: unidad y diferenciación. Revista Argentina de Educación, 1(2), 37-47.

Broström, S. (2006). Care and Education: Towards a New Paradigm in Early Childhood Education. Child and Youth Care Forum, 35(5-6), 391-409.

Cannella, G.S. (1997). Deconstructing Early Childhood Education: Social Justice and Revolution. New York: Peter Lang.

Clark, M.M. \& Waller, T. (2007). Early childhood education and care: policy and practice. Londres: Sage.

Cox, C. (1986). Políticas educacionales y principios culturales. Chile 1965-1985. Santiago de Chile: Centro de Investigación y Desarrollo de la Educación.

Dale, R. (1997). The state and the governance of education: an analysis of the restructuring of the state-education relationship. En A. Halsey, H. Lauder, P. Brown \& A. Stuart Wells (comps.), Education: Culture, Economy, and Society. Oxford: Oxford University Press.

Diker, G. (2002). Organización y perspectivas de la educación inicial en Iberoamérica principales tendencias. OEl. Recuperado de: www.oei.es/historico/linea3/diker.pdf

Ebbeck, M. \& Chan, Y.Y.Y. (2011). Instituting change in early childhood education: Recent developments in Singapore. Early Childhood Education Journal, 38(6), 457-463.

Fromberg, D.P. \& Williams, L.R. (2012). Encyclopedia of early childhood education. Nueva York: Routledge.

Gvirtz, S. (2002). Curricular reforms in Latin America with special emphasis on the Argentine case. Comparative Education, 38(4), 453-469.

Haft, H. \& Hopmann, S. (1990). Curriculum administration as symbolic action. En H. Haft \& S. Hopmann (comps.), Case studies in curriculum administration history. London: Falmer.

Irwin, L.G., Siddiqi, A. \& Hertzman, C. (2007). Early child development: A powerful equalizer. Final report to the WHO Commission on social determinants of health. Ginebra: OMS.

Kaga, Y., Bennett, J. \& Moss, P. (2010). Caring and learning together: A crossnational study on the integration of early childhood care and education within education. UNESCO.

Kramer, S. (2002). Propostas pedagógicas ou curriculares de educação infantil: para retomar o debate. Pro-Posições (Unicamp), 38(2), 65-82. 
Lohmander, M.K. \& Samuelsson, I.P. (2015). Play and learning in early childhood education in Sweden. Psychology in Russia, 8(2), 18-26.

Lourie, M. (2016). Bicultural education policy in New Zealand. Journal of Education Policy, 31(5), 637-650. doi: 10.1080/02680939.2016.1159339

Llobet, V. (2006). Las políticas sociales para la infancia vulnerable. Algunas reflexiones desde la Psicología. Revista Latinoamericana de Ciencias Sociales, Niñez y Juventud, 4(1), 149-176.

Machado, R.B. \& Lockmann, K. (2014). Base Nacional Comum, escola, professor. Revista Científica e-Curriculum, 12(3), 1591-1613.

Ministry of Education (1996). Te Whàriki, Early Childhood Curriculum. Wellington, New Zealand: Learning Media limited. Retrieved from http://www.educate.ece.govt.nz/ /media/educate/files/reference\%20downl oads/whariki.pdf

Morales, F. (2013). Radiografía de la institucionalidad para la primera infancia en Chile. Clave de políticas públicas. Desafíos en la Educación de Primera Infancia, 20, 1-9.

Moss, P. (2007). Meetings across the paradigmatic divide. Educational Philosophy and Theory, 39(3), 229-245.

Nores, M. \& Barnett, W.S. (2010). Benefits of early childhood interventions across the world: (Under) Investing in the very young. Economics of Education Review, 29(2), 271-282. doi: 10.1016/j.econedurev.2009.09.001

Peralta, M.V., Arancibia Lorca, E. \& Blanco Guijarro, R. (2004). Aprendiendo de las experiencias. Reforma Curricular de la Educación Parvularia. Santiago de Chile: MINEDUC-UNESCO.

Podmore, V. (2002). Politics in the Playground: The world of early childhood in postwar New Zealand. McGill Journal of Education, 37(1), 117.

Redondo, P. (2012). Políticas en debate, la atención educativa de la primera infancia en la Argentina. Propuesta educativa 37, 5-16.

Repetto, F., Langou, G.D., Aulicino, C., Achával, O.D. \& Acuña, M. (2016). El futuro es hoy. Primera infancia en la Argentina. Buenos Aires: Biblos-CIPPEC.

Rozengardt, A. (2014). Estudio sobre el rol de los servicios no formales de cuidado y educación de la primera infancia como dispositivos de inclusión social: propuesta de una matriz de valoración del papel de los espacios no formales de cuidado y educación de la primera infancia en la realización de los derechos humanos de las niñas y los niños. Tesis (Maestría en diseño y gestión de políticas y programas sociales). FLACSO, Buenos Aires. 
Taguma, M., Litjens, I., Makowiecki, K. \& Early, Q.M. (2012). Quality Matters in Early Childhood Education and Care: Sweden. París: OECD.

Terigi, F. (1999). Curriculum: Itinerarios para aprehender un territorio. Buenos Aires: Santillana.

Terigi, F. (2002). Análisis comparativo de los currículos iberoamericanos: procesos, condiciones y tensiones que debemos considerar. En IV Encuentro Internacional de Educación Inicial y Preescolar, La Habana, 2002. http://www.oei.es/linea3/TERIGI.pdf [09-10-15].

Ting, T.C. (2007). Policy developments in pre-school education in Singapore: A focus on the key reforms of kindergarten education. International Journal of Child Care and Education Policy, 1(1), 35-43.

Treviño, E., Valdés, H., Castro, M., Costilla, R., Pardo, C. \& Rivas, F.D. (2013). Factores asociados al logro cognitivo de los estudiantes de América Latina y el Caribe. Recuperado de:

http://unesdoc.unesco.org/images/0018/001867/186769S.pdf [10-01-16].

UNESCO. (2006). Strong foundations. Early childhood care and education: Education for All. París: UNESCO.

UNICEF. (2011). Crecer juntos para la primera infancia. Encuentro Regional de Políticas Integrales. Buenos Aires: UNICEF.

Veleda, C., Rivas, A. \& Mezzadra, F. (2011). La construcción de la justicia educativa: criterios de redestribución y reconocimiento para la educación argentina. Buenos Aires: CIPPEC.

Zibecchi, C. (2013). Organizaciones comunitarias y cuidado en la primera infancia: un análisis en torno a las trayectorias, prácticas y saberes de las cuidadoras. Trabajo y sociedad, 20, 427-447.

\section{Cómo citar este artículo:}

Guevara, J. \& Cardini, A. (2019). El lugar del currículum en la primera infancia: aportes de una mirada comparada. Profesorado. Revista de Currículum y Formación de Profesorado, 23(3), 473-491. DOI: 10.30827/profesorado.v23i3.11239 